\title{
МНОГОМЕРНОЕ НЕАВТОНОМНОЕ УРАВНЕНИЕ ВТОРОГО ПОРЯДКА СО СТЕПЕННЫМИ НЕЛИНЕЙНОСТЯМИ
}

\author{
И. В. Рахмелевич
}

(Статья представлена членом редакционной коллегии А. П. Солдатовым)

Нижегородский государственный университет им. Н. И. Лобачевского,

г. Нижний Новгород, 603950, Россия

E-mail: igor-kitpd@yandex.ru

\begin{abstract}
Аннотация. Исследовано неавтономное многомерное уравнение в частных производных второго порядка, правая часть которого содержит произвольную нелинейность по неизвестной функции и степенные нелинейности по её первым производным. Найдено решение этого уравнения типа бегущей волны в неявном виде. Для случая степенной нелинейности по неизвестной функции получены явные решения типа бегущей волны, в частности, в виде степенной, экспоненциальной и логарифмической функций. Также получены решения в виде квадратичного полинома и обобщённого монома, определены условия на параметры и правую часть уравнения, при которых данные решения существуют. Найдены частные решения, выраженные через функции от подмножеств независимых переменных, а также решения в виде линейной комбинации некоторых экспоненциальных функций. Проанализированы свойства найденных решений при различных параметрах уравнения.
\end{abstract}

Ключевые слова: нелинейность степенного типа, уравнение в частных производных с переменными коэффициентами, разделение переменных, решение типа бегущей волны.

Для цитирования: Рахмелевич И. В. 2020. Многомерное неавтономное уравнение второго порядка со степенными нелинейностями. Прикладная математика \& Физика, 52(2): 93-104.

DOI 10.18413/2687-0959-2020-52-2-93-104.

\section{MULTI-DIMENSIONAL NON-AUTONOMOUS SECOND ORDER EQUATION WITH POWER NONLINEARITIES}

\author{
I. V. Rakhmelevich \\ (Article submitted by a member of the editorial board A. P. Soldatov) \\ Nizhniy Novgorod State University, \\ Nizhniy Novgorod, 603950, Russia \\ E-mail: igor-kitpd@yandex.ru \\ Received May 12, 2020
}

\begin{abstract}
We consider non-autonomous multi-dimensional partial differential equation of the second order, the right side of which contains arbitrary nonlinearity on the unknown function and power nonlinearities on its first partial derivatives. There is founded the solution of travelling wave type for this equation in the implicit form. There are received the explicit solutions of travelling wave type for the case of power nonlinearity on the unknown function. In particular, these solutions can be in the form of some elementary functions. There are also received the solutions in the form of quadratic polynomial and generalized monomial, and the conditions on the parameters and on the right side of equation are determined, under which these solutions exist. There are founded some solutions, which can be represented through the functions of independent variables subsets, in particular, in the form of sum and production of such functions, and the solutions of aggregated travelling wave type. Also there are received the solutions in the form of linear combination of some exponential functions. The properties of the founded solutions under the different parameters of equation are analysed.
\end{abstract}

Key words: power type nonlinearity, partial differential equation with variable coefficients, separation of variables, travelling wave type solution.

For citation: Rakhmelevich I. V. 2020. Multi-dimensional non-autonomous second order equation with power nonlinearities. Applied Mathematics \& Physics, 52(2): 93-104 (in Russian). DOI 10.18413/2687-0959-2020-52-293-104.

Введение. В современной математической физике большое внимание уделяется исследованию решений уравнений в частных производных с различными типами нелинейностей. В частности, 
результаты по уравнениям с нелинейностями степенного типа широко представлены как в известных пособиях и справочниках [Полянин, Зайцев, 2002], [Кудряшов, 2010], так и в оригинальных работах [Рахмелевич, 2016 б; 2017 а, б; 2018], [Zhdanov, 1994], [Grundland, Infeld, 1992], [Polyanin, 2019 a, b]. Так, результаты исследования двумерных гиперболических и эллиптических уравнений приведены в работах [Рахмелевич, 2017б,в], [Miller, Rubel, 1993]. При этом одним из наиболее эффективных является метод разделения переменных [Полянин, Зайцев, Журов, 2005]; также широко применяются методы аналитической теории дифференциальных уравнений [Кудряшов, 2010], методы группового анализа, метод дифференциальных связей, метод Кларксона - Крускала и другие [Polyanin, 2019 b]. В последние годы много работ посвящено исследованию неавтономных линейных и нелинейных уравнений [Рахмелевич, 2016б, 2018], [Polyanin, 2019 a, b]. Целью данной работы является исследование некоторых классов решений неавтономного уравнения в частных производных второго порядка, правая часть которого содержит степенные нелинейности по искомой функции и её первым производным.

1. Исходное уравнение и его простейшие решения. Рассмотрим уравнение в частных производных относительно неизвестной функции $u(X)$ :

$$
\sum_{i=1}^{N} a_{i} \frac{\partial^{2} u}{\partial x_{i}^{2}}=g(u) F(X) \prod_{i=1}^{N}\left(\frac{\partial u}{\partial x_{i}}\right)^{\beta_{i}}
$$

$a_{i}, \beta_{i}$ - вещественные параметры, $g(u), F(X)$ - заданные функции. Здесь и далее будут использоваться обозначения: $X=\left\{x_{1}, \ldots, x_{N}\right\}$ - множество независимых переменных; $I=\{1, \ldots, N\}-$ множество значений индекса, нумерующего независимые переменные.

Следующая теорема определяет решения для уравнения (1.1), зависящие от линейной комбинации независимых переменных.

Теорема 1.1. Пусть $F(X) \equiv 1$. Тогда уравнение (1.1) имеет решение типа бегущей волны, которое может быть представлено в неявном виде:

1) при $\beta_{\Sigma}=2$ :

$$
\sum_{n=1}^{N} c_{n} x_{n}-z_{0}=V_{0} \int \exp (-C G(u)) d u
$$

2) $n p u \beta_{\Sigma} \neq 2$ :

$$
\sum_{n=1}^{N} c_{n} x_{n}-z_{0}=\left(2-\beta_{\Sigma}\right)^{\frac{1}{\beta_{\Sigma}-2}} \int(C G(u)+A)^{\frac{1}{\beta_{\Sigma}^{-2}}} d u
$$

В формулах (1.2), (1.3) $c_{n}, z_{0}, V_{0}, A$ - произвольные постоянные; $\beta_{\Sigma}, C, G(u)$ определяются выражениями:

$$
\begin{gathered}
\beta_{\Sigma}=\sum_{i=1}^{N} \beta_{i}, \quad C=\frac{\prod_{i=1}^{N} c_{i}^{\beta_{i}}}{\sum_{i=1}^{N} a_{i} c_{i}^{2}}, \\
G(u)=\int g(u) d u
\end{gathered}
$$

причём постоянные $c_{i}$ должсны удовлетворять условию:

$$
\sum_{i=1}^{N} a_{i} c_{i}^{2} \neq 0
$$

Доказательство. Решение уравнения (1.1) ищем в виде:

$$
u(X)=U(z), \quad z=\sum_{n=1}^{N} c_{n} x_{n} .
$$

Подставляя (1.6) в уравнение (1.1), получаем уравнение:

$$
U^{\prime \prime}(z) \sum_{i=1}^{N} a_{i} c_{i}^{2}=g(U)\left[U^{\prime}(z)\right]^{\beta_{\Sigma}} \prod_{i=1}^{N} c_{i}^{\beta_{i}}
$$

Учитывая условие (1.5) и вводя функцию $G(U)$ согласно (1.4б), уравнение $(1.7)$ преобразуется так:

$$
U^{\prime \prime}(z)\left[U^{\prime}(z)\right]^{1-\beta_{\Sigma}}=C \frac{d}{d z} G(U)
$$


Проанализируем уравнение (1.8) для случаев, перечисленных в условии теоремы.

1. При $\beta_{\Sigma}=2$ уравнение (1.8) запишется в виде:

$$
\frac{U^{\prime \prime}(z)}{U^{\prime}(z)}=C \frac{d}{d z} G(U)
$$

В результате интегрирования (1.9) сводится к уравнению первого порядка:

$$
\ln \left|U^{\prime}(z)\right|=C G(U)-\ln V_{0} .
$$

Разделяя переменные и интегрируя уравнение (1.10), получаем:

$$
z-z_{0}=V_{0} \int \exp (-C G(U)) d U .
$$

Учитывая (1.6), из (1.11) получаем решение в виде (1.2).

2. При $\beta_{\Sigma} \neq 2$ уравнение (1.8) преобразуем к виду:

$$
\frac{d}{d z}\left\{\left[U^{\prime}(z)\right]^{2-\beta_{\Sigma}}-\left(2-\beta_{\Sigma}\right) C G(U)\right\}=0 .
$$

В результате интегрирования (1.12) получаем:

$$
U^{\prime}(z)=\left\{\left(2-\beta_{\Sigma}\right)(C G(U)+A)\right\}^{\frac{1}{2-\beta_{\Sigma}}} .
$$

Интегрируя (1.13), находим:

$$
z-z_{0}=\left(2-\beta_{\Sigma}\right)^{\frac{1}{\beta_{\Sigma}-2}} \int(C G(U)+A)^{\frac{1}{\beta_{\Sigma}-2}} d U
$$

Используя (1.6), получаем из (1.14) для данного случая решение в виде (1.3). Теорема доказана.

Рассмотрим теперь решения уравнения (1.1) типа бегущей волны в случае, когда $F(X)=f(z)$, причём $z$ определяется выражением (1.6).

1. Пусть функции $F(X), g(u)$ определяются выражениями:

$$
F(X)=\exp (\alpha z), \quad g(u)=g_{0} u^{\gamma}
$$

где $g_{0}, \alpha, \gamma-$ вещественные параметры. Тогда предполагаемый вид решения уравнения (1.1) будет следующим:

$$
u=U_{0} \exp (z)
$$

где $U_{0}$ - неизвестная постоянная, подлежащая определению в дальнейшем. Подставив (1.16) в (1.1) и учитывая (1.15), находим:

$$
U_{0} \exp (z) \sum_{i=1}^{N} a_{i} c_{i}^{2}=g_{0} U_{0}^{\beta_{\Sigma}+\gamma} \exp \left(\left(\alpha+\beta_{\Sigma}+\gamma\right) z\right) \prod_{i=1}^{N} c_{i}^{\beta_{i}} .
$$

Предполагая, что постоянные $c_{i}$ удовлетворяют условию (1.5), и выполняя элементарные преобразования, из (1.17) получаем:

$$
U_{0}^{1-\beta_{\Sigma}-\gamma}=g_{0} C \exp \left(\left(\alpha+\beta_{\Sigma}+\gamma-1\right) z\right)
$$

где $C$ определяется выражением (1.4а). Уравнение (1.18) можно удовлетворить только в том случае, если параметры удовлетворяют условию:

$$
\alpha+\beta_{\Sigma}+\gamma=1
$$

Предполагая, что условие (1.19) выполняется, из (1.18) находим:

а) если $\beta_{\Sigma}+\gamma \neq 1$ :

$$
U_{0}=\left(g_{0} C\right)^{\frac{1}{1-\beta_{\Sigma}-\gamma}},
$$

б) если $\beta_{\Sigma}+\gamma=1$, то $U_{0}-$ произвольная, а постоянные $c_{i}$ должны удовлетворять условию:

$$
g_{0} C=1
$$


2. Пусть функции $F(X), g(u)$ определяются выражениями:

$$
F(X)=z^{\alpha}, \quad g(u)=g_{0} u^{\gamma}
$$

Тогда предполагаем следующий вид решения уравнения (1.1):

$$
u=U_{0} z^{\sigma}
$$

где $U_{0}, \sigma$ - неизвестные постоянные, подлежащие определению. Подставив (1.22) в (1.1), откуда с учётом (1.21) и (1.5) следует:

$$
\sigma^{1-\beta_{\Sigma}}(\sigma-1) z^{\sigma\left(1-\beta_{\Sigma}-\gamma\right)+\beta_{\Sigma}-\alpha-2}=g_{0} C U_{0}^{\beta_{\Sigma}+\gamma-1} .
$$

Уравнение (1.23) можно удовлетворить, если выполнено условие:

$$
\sigma\left(\beta_{\Sigma}+\gamma-1\right)+\alpha-\beta_{\Sigma}+2=0 .
$$

a) если $\beta_{\Sigma}+\gamma \neq 1$, то из $(1.23),(1.24)$ следует, что $U_{0}, \sigma$ определяются выражениями:

$$
\sigma=\frac{\beta_{\Sigma}-\alpha-2}{\beta_{\Sigma}+\gamma-1}, \quad U_{0}=\left(\frac{g_{0} C}{\sigma^{1-\beta_{\Sigma}}(\sigma-1)}\right)^{\frac{1}{1-\beta_{\Sigma}-\gamma}}
$$

Если, кроме того, $\alpha+\gamma=-1$, то из (1.25) получаем $\sigma=1$. Тогда из выражения $(1.25)$ для $U_{0}$ следует, что при $\beta_{\Sigma}+\gamma<1$ решение вида (1.22) не существует, а при $\beta_{\Sigma}+\gamma>1 U_{0}=0$ - решение вырождается в тривиальное.

б) если $\beta_{\Sigma}+\gamma=1$, то из (1.23), (1.24) следует, что $U_{0}$ - произвольная; причём решение $(1.22)$ существует только при дополнительном условии:

$$
\beta_{\Sigma}-\alpha-2=0
$$

при этом $\sigma$ должно удовлетворять уравнению:

$$
\sigma^{1-\beta_{\Sigma}}(\sigma-1)=g_{0} C
$$

3. Пусть выполнены условия (1.21), причём $\gamma=0$. Покажем, что в этом случае уравнение (1.1) может иметь логарифмическое решение:

$$
u=U_{0} \ln |z| .
$$

Подставив (1.28) в (1.1) и с учётом (1.21), (1.5), имеем:

$$
U_{0}^{1-\beta_{\Sigma}}=-g_{0} C z^{\alpha-\beta_{\Sigma}+2} .
$$

Это уравнение можно удовлетворить только в том случае, если выполнено условие (1.26).

а) если $\beta_{\Sigma} \neq 1$, то из (1.29) следует, что $U_{0}$ определяется выражением:

$$
U_{0}=\left(-g_{0} C\right)^{\frac{1}{1-\beta_{\Sigma}}}
$$

б) если $\beta_{\Sigma}=1$, то из (1.29) следует, что $U_{0}$ - произвольная; причём решение (1.28) существует только при дополнительном условии:

$$
g_{0} C=-1
$$

Итак, в результате проведённых выше рассуждений доказана следующая теорема:

Теорема 1.2. 1. Пусть функиии $F(X), g(u)$ определяются выражениями (1.15), где z определяется второй формулой (1.6). Всюду в данной теореме предполагается, что коэффициенты с $c_{i}$ удовлетворяют условию (1.5). Пусть также параметры уравнения (1.1) удовлетворяют условию (1.19). Тогда уравнение (1.1) имеет экспоненциалъное решение (1.16), причём:

а) если $\beta_{\Sigma}+\gamma \neq 1$, то постоянная $U_{0}$ определяется выражением (1.20а);

б) если $\beta_{\Sigma}+\gamma=1$, то $U_{0}$ - произвольная, а решение (1.16) существует при дополнительном условии (1.20б).

2. Пусть функиии $F(X), g(u)$ определяются выражениями (1.21). Тогда уравнение (1.1) имеет степенное решение (1.22), причём:

а) если $\beta_{\Sigma}+\gamma \neq 1$, то $U_{0}, \sigma$ определяются выражениями (1.25);

б) если $\beta_{\Sigma}+\gamma=1$, то $U_{0}-$ произвольнал, а б должно удовлетворять уравнению (1.27); при этом решение (1.22) существует толъко при дополнительном условии (1.26). 
3. Пусть функиии $F(X), g(u)$ определяются выражениями (1.21), где $\gamma=0$, а также удовлетворлется условие (1.26). Тогда уравнение (1.1) имеет логарифмическое решение (1.28), причём:

а) если $\beta_{\Sigma} \neq 1$, то $U_{0}$ определяется выражением (1.30);

б) если $\beta_{\Sigma}=1$, то $U_{0}$ - произвольнал; а решение (1.28) существует только при дополнительном условии (1.31).

Теорема 1.3. Пусть функиии $F(X), g(u)$ определяются выражениями:

$$
F(X)=\prod_{i=1}^{N}\left(p_{i} x_{i}+q_{i}\right)^{-\beta_{i}}, \quad g(u)=g_{0},
$$

где $p_{i}, q_{i}$ - вещественные параметры. Тогда уравнение (1.1) имеет решение в виде квадратичного полинома:

$$
u(X)=U_{0} \sum_{n=1}^{N}\left(\frac{p_{n} x_{n}^{2}}{2}+q_{n} x_{n}\right),
$$

причём:

а) если $\beta_{\Sigma} \neq 1$, то $U_{0}$ выражается так:

$$
U_{0}=\left(\frac{1}{g_{0}} \sum_{i=1}^{N} a_{i} p_{i}\right)^{\frac{1}{\beta_{\Sigma}-1}}
$$

б) если $\beta_{\Sigma}=1$, то $U_{0}$ - произвольная, при этом необходимо, чтобы параметры уравнения удовлетворяли условию:

$$
\sum_{i=1}^{N} a_{i} p_{i}=g_{0}
$$

Доказательство. Подставляя (1.33) в уравнение (1.1) и учитывая (1.32), получаем:

$$
U_{0}^{\beta_{\Sigma}-1}=\frac{1}{g_{0}} \sum_{i=1}^{N} a_{i} p_{i} .
$$

При $\beta_{\Sigma} \neq 1$ из (1.35) следует, что функция (1.33) является решением уравнения (1.1), если $U_{0}$ определяется выражением (1.34а). В случае $\beta_{\Sigma}=1$ уравнение (1.35) сводится к (1.34б), а постоянная $U_{0}$ может быть произвольной. Теорема доказана.

Теорема 1.4. Пусть функиии $F(X), g(u)$ определяются выражениями:

$$
F(X)=\sum_{i=1}^{N} \frac{b_{i}}{x_{i}^{2}} \prod_{i=1}^{N} x_{i}^{\alpha_{i}}, \quad g(u)=g_{0} u^{\gamma} .
$$

Тогда уравнение (1.1) имеет решение в виде обобщённого монома:

$$
u(X)=U_{0} \prod_{n=1}^{N} x_{n}^{\sigma_{n}} .
$$

При этом возможны два случая:

1) если $\beta_{\Sigma}+\gamma \neq 1$, то $\sigma_{n}, U_{0}$ определяются выражениями:

$$
\sigma_{n}=\frac{\beta_{n}-\alpha_{n}}{\beta_{\Sigma}+\gamma-1}, \quad U_{0}=\left(g_{0} \prod_{n=1}^{N} \sigma_{n}^{\beta_{n}}\right)^{\frac{1}{1-\beta_{\Sigma}-\gamma}} ;
$$

2) если $\beta_{\Sigma}+\gamma=1$, то решение (1.37) существует, если при $\forall n \in I \beta_{n}=\alpha_{n}$. Тогда $\sigma_{n}, U_{0}$ являются произвольными, но при этом $\sigma_{n}$ должны удовлетворлть условию:

$$
g_{0} \prod_{n=1}^{N} \sigma_{n}^{\beta_{n}}=1 .
$$

Кроме того, во всех перечисленных выше случалх при $\forall n \in I$ должны быть выполнены условия:

$$
b_{n}=a_{n} \sigma_{n}\left(\sigma_{n}-1\right)
$$


Доказательство. Подставляя (1.37) в уравнение (1.1), получаем:

$$
\sum_{i=1}^{N} \frac{a_{i} \sigma_{i}\left(\sigma_{i}-1\right)}{x_{i}^{2}}=g_{0}[u(X)]^{\beta_{\Sigma}+\gamma-1} \sum_{i=1}^{N} \frac{b_{i}}{x_{i}^{2}} \prod_{n=1}^{N} \sigma_{n}^{\beta_{n}} x_{n}^{\alpha_{n}-\beta_{n}} .
$$

Уравнение (1.41) можно удовлетворить, если при $\forall n \in I$ выполняются условия:

$$
\sigma_{n}\left(\beta_{\Sigma}+\gamma-1\right)+\left(\alpha_{n}-\beta_{n}\right)=0,
$$

а также условия (1.40).

Если $\beta_{\Sigma}+\gamma \neq 1$, то из (1.42) следует, что $\sigma_{n}$ определяется первым из выражений (1.38); из (1.41) следует, что $U_{0}$ определяется вторым из выражений (1.38). Если же $\beta_{\Sigma}+\gamma=1$, то условия (1.42) выполняются, если при $\forall n \in I \beta_{n}=\alpha_{n}$; при этом $\sigma_{n}$ могут быть произвольными. Также из (1.41) в этом случае следует, что $U_{0}$ являются произвольной, при этом необходимо, чтобы $\sigma_{n}$ удовлетворяли условию (1.39). Доказательство закончено.

Пусть теперь предполагаемый вид решения определяется формулой:

$$
u(X)=U(z), \quad z=\sum_{n=1}^{N} \varphi_{n}\left(x_{n}\right),
$$

где $\varphi_{n}\left(x_{n}\right)$ - некоторые функции, которые будут определены ниже. Используя (1.43), уравнение (1.1) можно записать в виде:

$$
U^{\prime \prime}(z) \sum_{i=1}^{N} a_{i}\left[\varphi_{i}^{\prime}\left(x_{i}\right)\right]^{2}+U^{\prime}(z) \sum_{i=1}^{N} a_{i} \varphi_{i}^{\prime \prime}\left(x_{i}\right)=g(U) F(X)\left[U^{\prime}(z)\right]^{\beta_{\Sigma}} \prod_{i=1}^{N}\left[\varphi_{i}^{\prime}\left(x_{i}\right)\right]^{\beta_{i}},
$$

Предположим, что функции $\varphi_{i}\left(x_{i}\right)$ таковы, что выполняется соотношение:

$$
\sum_{i=1}^{N} a_{i} \varphi_{i}^{\prime \prime}\left(x_{i}\right)=P=\text { const. }
$$

Из (1.45) следует, что в этом случае $\varphi_{i}\left(x_{i}\right)$ представляют собой квадратичные функции:

$$
\varphi_{i}\left(x_{i}\right)=\frac{p_{i} x_{i}^{2}}{2}+q_{i} x_{i}+r_{i} .
$$

Тогда уравнение (1.44) может быть сведено к обыкновенному дифференциальному уравнению (ОДУ) относительно $U(z)$, если выполнены условия:

$$
a_{i}\left[\varphi_{i}^{\prime}\left(x_{i}\right)\right]^{2}=\lambda \varphi_{i}\left(x_{i}\right), \quad F(X)=\Phi(z) \prod_{i=1}^{N}\left[\varphi_{i}^{\prime}\left(x_{i}\right)\right]^{-\beta_{i}}
$$

где $\Phi(z)$ - некоторая заданная функция; $\lambda$ - вещественная постоянная. Тогда, подставляя (1.46) в первое из условий (1.47), находим:

$$
p_{i}=\frac{\lambda}{2 a_{i}}, \quad r_{i}=\frac{a_{i} q_{i}^{2}}{\lambda}
$$

В свою очередь, из (1.45) и (1.48) следует:

$$
\sum_{i=1}^{N} a_{i} \varphi_{i}^{\prime \prime}\left(x_{i}\right)=\frac{N \lambda}{2}
$$

Тогда из (1.44) с учётом (1.47) и (1.49), получаем, что функция $U(z)$ должна удовлетворять следующему ОДУ:

$$
z U^{\prime \prime}(z)+\frac{N}{2} U^{\prime}(z)=\frac{g(U)}{\lambda}\left[U^{\prime}(z)\right]^{\beta_{\Sigma}} \Phi(z) .
$$

Таким образом, в результате проведённых выше рассуждений доказана следующая теорема:

Теорема 1.5. Пусть функиия $F(X)$ определяетсл выражением:

$$
F(X)=\Phi(z) \prod_{i=1}^{N}\left(p_{i} x_{i}+q_{i}\right)^{-\beta_{i}}, \quad z=\sum_{i=1}^{N}\left(\frac{p_{i} x_{i}^{2}}{2}+q_{i} x_{i}+r_{i}\right) .
$$


Здесь $\Phi(z)$ - некоторая заданная функиия; $\lambda, q_{i}$ - вещественные постоянные; $p_{i}, r_{i}$ выражаются по формулам (1.48). Тогда уравнение (1.1) имеет решение вида $u=U(z)$, причём $U(z)$ находится из уравнения (1.50).

2. Решения, зависящие от подмножеств независимых переменных. Пусть множество значений $I=\{1, \ldots, N\}$ индекса $n$, нумерующего независимые переменные, представлено в виде объединения $K$ подмножеств $I_{l}(l=1, \ldots, K)$, причём $I_{l_{1}} \cap I_{l_{2}}=\oslash$ при любых $l_{1} \neq l_{2}$. Тогда множество переменных $X=\left\{x_{1}, x_{2}, \ldots, x_{N}\right\}$ также может быть представлено в виде объединения $K$ непересекающихся подмножеств $X_{l}=\left\{x_{n}\right\}_{n \in I_{l}}$. Также здесь и далее всюду будем обозначать $\Xi=\{1, \ldots, K\}-$ множество значений индекса $l$.

Агрегированными переменными будем называть переменные вида: $y_{l}=\sum_{n \in I_{l}} \varphi_{n}\left(x_{n}\right)$, где $\varphi_{n}\left(x_{n}\right)-$ некоторые заданные функции. Данный параграф посвящён исследованию решений, зависящих от функций некоторых подмножеств независимых переменных, и в частности, от агрегированных переменных. В простейшем случае агрегированные переменные представляют собой линейные комбинации исходных независимых переменных: $y_{l}=\sum_{n \in I_{l}} c_{n} x_{n}$.

Теорема 2.1. Пусть функиия $F(X)$ определяется выражением:

$$
F(X)=\exp \left(-\sum_{l=1}^{K} \alpha_{l} \sum_{n \in I_{l}} c_{n} x_{n}\right)
$$

1. Пусть также $k \in \Xi-$ некоторое выбранное фиксированное значение, причём выполнены условия:

$$
\begin{gathered}
A_{l} \equiv \sum_{i \in I_{l}} a_{i} c_{i}^{2}=0 \quad(\forall l \neq k) \\
\alpha_{l}=\beta_{\Sigma l} \quad(\forall l \neq k), \quad \alpha_{k}=\beta_{\Sigma k}-1, \quad \beta_{\Sigma l}=\sum_{i \in I_{l}} \beta_{i}, \\
g(u)=g_{0} .
\end{gathered}
$$

Тогда уравнение (1.1) имеет решение вида:

$$
u(X)=\sum_{l=1}^{K} B_{l} \exp \left(\sum_{n \in I_{l}} c_{n} x_{n}\right)
$$

где коэффициенты $B_{l}$ связаны соотношением:

$$
g_{0} \prod_{l=1}^{K} P_{l} B_{l}^{\beta_{\Sigma l}}=A_{k} B_{k}, \quad P_{l}=\prod_{i \in I_{l}} c_{i}^{\beta_{i}}
$$

2. Пусть при $\forall l \in \Xi$ выполнены условия:

$$
A_{l}=A \neq 0, \quad \alpha_{l}=\beta_{\Sigma l}, \quad g(u)=g_{0} u .
$$

Тогда уравнение (1.1) имеет решение вида (2.3), причём коэффициенты $B_{l}$ связаны соотношением:

$$
g_{0} \prod_{l=1}^{K} P_{l} B_{l}^{\beta_{\Sigma l}}=A
$$

Доказательство. 1. Подставляя (2.3) в уравнение (1.1), с учётом (2.2в) получаем:

$$
\sum_{l=1}^{K} A_{l} B_{l} \exp \left(\sum_{n \in I_{l}} c_{n} x_{n}\right)=g_{0} \prod_{l=1}^{K} P_{l} B_{l}^{\beta_{\Sigma l}} \exp \left(\left(\beta_{\Sigma l}-\alpha_{l}\right) \sum_{n \in I_{l}} c_{n} x_{n}\right) .
$$

Пусть выбрано такое $k \in \Xi$, что при $\forall l \neq k$ выполнены условия (2.2a). Тогда уравнение (2.7) можно привести к виду:

$$
\frac{g_{0} P_{k}}{A_{k}} B_{k}^{\beta_{\Sigma k}-1} \exp \left(\left(\beta_{\Sigma k}-\alpha_{k}\right) \sum_{n \in I_{k}} c_{n} x_{n}\right) \prod_{l \in \Xi_{k}^{-}} P_{l} B_{l}^{\beta_{\Sigma l}} \exp \left(\left(\beta_{\Sigma l}-\alpha_{l}\right) \sum_{n \in I_{l}} c_{n} x_{n}\right)=1 .
$$


Здесь использовано обозначение $\Xi_{k}^{-}=\Xi \backslash\{k\}$. Уравнение (2.8) может удовлетворяться, если выполнены условия (2.2б). Тогда оно сводится к следующему:

$$
\frac{g_{0}}{A_{k} B_{k}} \prod_{l=1}^{K} P_{l} B_{l}^{\beta_{\Sigma l}}=1
$$

откуда получаем соотношение (2.4).

2. Аналогичным образом, в случае, когда выполнены условия (2.5), подставляя (2.3) в уравнение (1.1), после некоторых преобразований получаем:

$$
g_{0} \prod_{l=1}^{K} P_{l} B_{l}^{\beta_{\Sigma l}} \exp \left(\left(\beta_{\Sigma l}-\alpha_{l}\right) \sum_{n \in I_{l}} c_{n} x_{n}\right)=A .
$$

Уравнение (2.9) можно удовлетворить, если при $\forall l \in \Xi$ выполнено второе из условий (2.5). Тогда из (2.9) непосредственно следует соотношение (2.6). Теорема доказана.

Теорема 2.2. Пусть функиии $g(u), F(X)$ удовлетворяют соотношениям:

$$
g(u)=g_{0} \exp (\lambda u), \quad F(X)=\prod_{l=1}^{K} f_{l}\left(X_{l}\right)
$$

где $f_{l}\left(X_{l}\right)$ - некоторые заданные функции. Тогда уравнение (1.1) имеет решение вида

$$
u(X)=\sum_{l=1}^{K} u_{l}\left(X_{l}\right)
$$

в следующих случалх:

1. Если при $\forall l \in \Xi$ функции $u_{l}\left(X_{l}\right)$ удовлетворяют переопределённой системе уравнений:

$$
\sum_{i \in I_{l}} a_{i} \frac{\partial^{2} u_{l}}{\partial x_{i}^{2}}=p_{l}, \quad f_{l}\left(X_{l}\right) \exp \left(\lambda u_{l}\left(X_{l}\right)\right) \prod_{i \in I_{l}}\left(\frac{\partial u_{l}}{\partial x_{i}}\right)^{\beta_{i}}=q_{l},
$$

где $p_{l}, q_{l}$ - постолнные, удовлетворяющие условию:

$$
\sum_{l=1}^{K} p_{l}=g_{0} \prod_{l=1}^{K} q_{l}
$$

2.Если $n p и \forall l \in \Xi, \quad l \neq k$ функции $u_{l}\left(X_{l}\right)$ удовлетворяют переопределённой системе уравнений (2.11), а функиия $u_{k}\left(X_{k}\right)$ удовлетворяет уравнению:

$$
\sum_{i \in I_{k}} a_{i} \frac{\partial^{2} u_{k}}{\partial x_{i}^{2}}=g_{0} \tilde{q}_{k} f_{k}\left(X_{k}\right) \prod_{i \in I_{k}}\left(\frac{\partial u_{k}}{\partial x_{i}}\right)^{\beta_{i}}-\tilde{p}_{k},
$$

әде

$$
\tilde{p}_{k}=\sum_{l \in \Xi_{k}^{-}} p_{l}, \quad \tilde{q}_{k}=\prod_{l \in \Xi_{k}^{-}} q_{l}, \quad \Xi_{k}^{-}=\Xi \backslash\{k\} .
$$

Здесь $k \in \Xi-$ некоторое выбранное фиксированное значение.

Доказательство. Подставляя (2.11) в уравнение (1.1) и учитывая (2.10), получаем:

$$
\sum_{l=1}^{K} \hat{M}_{l} u_{l}\left(X_{l}\right)=g_{0} \prod_{l=1}^{K} Q_{l}\left(X_{l}, u_{l}\right)
$$

Здесь введены обозначения:

$$
\hat{M}_{l}=\sum_{i \in I_{l}} a_{i} \frac{\partial^{2}}{\partial x_{i}^{2}}, \quad Q_{l}\left(X_{l}, u_{l}\right)=f_{l}\left(X_{l}\right) \exp \left(\lambda u_{l}\left(X_{l}\right)\right) \prod_{i \in I_{l}}\left(\frac{\partial u_{l}}{\partial x_{i}}\right)^{\beta_{i}} .
$$

Для уравнения (2.15) возможны два случая.

1. При $\forall l \in \Xi$ функции $u_{l}\left(X_{l}\right)$ удовлетворяют уравнениям:

$$
Q_{l}\left(X_{l}, u_{l}\right)=q_{l} .
$$


Тогда, подставляя (2.17) в (2.15), находим:

$$
\sum_{l=1}^{K} \hat{M}_{l} u_{l}\left(X_{l}\right)=g_{0} \prod_{l=1}^{K} q_{l}
$$

Правая часть уравнения (2.18) является константой, а левая часть представлена в виде суммы функций от разных переменных, поэтому функции $u_{l}\left(X_{l}\right)$ должны удовлетворять также уравнениям:

$$
\hat{M}_{l} u_{l}\left(X_{l}\right)=p_{l} \text {. }
$$

Из (2.17) и (2.19) с учётом (2.16) следует, что при $\forall l \in \Xi$ функции $u_{l}\left(X_{l}\right)$ должны удовлетворять системе (2.12). Подставив (2.19) в (2.18), получаем условие $(2.13)$ для постоянных $p_{l}, q_{l}$.

2. При некотором $k \in \Xi Q_{k}\left(X_{k}, u_{k}\right) \neq$ const. Тогда существует такое $j \in I_{k}$, что $\frac{\partial Q_{k}}{\partial x_{j}} \neq 0$. Продифференцируем уравнение $(2.15)$ по $x_{j}$ и почленно разделив на $\frac{\partial Q_{k}}{\partial x_{j}}$, в результате получаем:

$$
\frac{\partial}{\partial x_{j}}\left\{\hat{M}_{k} u_{k}\left(X_{k}\right)\right\}\left\{\frac{\partial}{\partial x_{j}} Q_{k}\left(X_{k}, u_{k}\right)\right\}^{-1}=g_{0} \prod_{l=1, l \neq k}^{K} Q_{l}\left(X_{l}, u_{l}\right) .
$$

Так как левая часть уравнения (2.20) содержит только переменные $X_{k}$, а правая часть - только переменные $X_{l}(l \neq k)$, то при $\forall l \in \Xi, \quad l \neq k$ функции $u_{l}\left(X_{l}\right)$ должны удовлетворять уравнению (2.17). Рассуждая аналогично случаю 1 , получаем, что эти функции должны также удовлетворять уравнению (2.19). Тогда, с учётом (2.17) и (2.19), уравнение (2.15) можно переписать в виде:

$$
\hat{M}_{k} u_{k}\left(X_{k}\right)+\sum_{l=1, l \neq k}^{K} p_{l}=g_{0} Q_{k}\left(X_{k}, u_{k}\right) \prod_{l=1, l \neq k}^{K} q_{l} .
$$

Принимая во внимание (2.14a) и $(2.16)$, получаем, что функция $u_{k}\left(X_{k}\right)$ должна удовлетворять уравнению (2.14). Теорема доказана.

Теорема 2.3. Пусть функции $g(u), F(X)$ удовлетворяют соотношениям:

$$
g(u)=g_{0} u^{\gamma}, \quad F(X)=\prod_{l=1}^{K} f_{l}\left(X_{l}\right)
$$

где $f_{l}\left(X_{l}\right)$ - некоторые заданные функции. Тогда уравнение (1.1) имеет решение вида

$$
u(X)=\prod_{l=1}^{K} u_{l}\left(X_{l}\right)
$$

в следующих случалх:

1. Если при $\forall l \in \Xi ф у н к ц и и ~ u_{l}\left(X_{l}\right)$ удовлетворяют переопределённой системе уравнений:

$$
\sum_{i \in I_{l}} a_{i} \frac{\partial^{2} u_{l}}{\partial x_{i}^{2}}=p_{l} u_{l}\left(X_{l}\right), \quad f_{l}\left(X_{l}\right)\left[u_{l}\left(X_{l}\right)\right]^{\beta_{\Sigma}+\gamma-1-\beta_{\Sigma l}} \prod_{i \in I_{l}}\left(\frac{\partial u_{l}}{\partial x_{i}}\right)^{\beta_{i}}=q_{l},
$$

где $p_{l}, q_{l}$ - постолнные, удовлетворяющие условию:

$$
\sum_{l=1}^{K} p_{l}=g_{0} \prod_{l=1}^{K} q_{l}
$$

2.Если при $\forall l \in \Xi, \quad l \neq k$ функиии $u_{l}\left(X_{l}\right)$ удовлетворяют переопределённой системе уравнений (2.24), а функиия $u_{k}\left(X_{k}\right)$ удовлетворяет уравнению:

$$
\sum_{i \in I_{k}} a_{i} \frac{\partial^{2} u_{k}}{\partial x_{i}^{2}}=g_{0} \tilde{q}_{k} f_{k}\left(X_{k}\right)\left[u_{k}\left(X_{k}\right)\right]^{\beta_{\Sigma}+\gamma-\beta_{\Sigma k}} \prod_{i \in I_{k}}\left(\frac{\partial u_{k}}{\partial x_{i}}\right)^{\beta_{i}}-\tilde{p}_{k} u_{k}\left(X_{k}\right),
$$

где $\tilde{p}_{k}, \tilde{q}_{k}$ определяются выражениями (2.14а).

Доказательство. Подставляя (2.23) в уравнение (1.1) и учитывая (2.22), получаем:

$$
\sum_{l=1}^{K} \frac{1}{u_{l}\left(X_{l}\right)} \hat{M}_{l} u_{l}\left(X_{l}\right)=g_{0} \prod_{l=1}^{K} Q_{l}\left(X_{l}, u_{l}\right)
$$


В этом уравнении $Q_{l}\left(X_{l}, u_{l}\right)$ определяется выражением:

$$
Q_{l}\left(X_{l}, u_{l}\right)=f_{l}\left(X_{l}\right)\left[u_{l}\left(X_{l}\right)\right]^{\beta_{\Sigma}+\gamma-1-\beta_{\Sigma l}} \prod_{i \in I_{l}}\left(\frac{\partial u_{l}}{\partial x_{i}}\right)^{\beta_{i}}
$$

а $\hat{M}_{l}$ определяется выражением (2.16). Проводя для уравнения (2.27) с учётом (2.28) рассуждения, полностью аналогичные доказательству теоремы 2.2 , получаем систему $(2.24)$ с дополнительным условием (2.25) и уравнение (2.26). Теорема доказана.

Следующая теорема определяет возможность редукции уравнения (1.1) к уравнению меньшей размерности $K<N$ с использованием переменных бегущей волны; аналогичные процедуры редукции применялись в работах [Кудряшов, 2010], [Полянин, Зайцев, Журов, 2005], [Рахмелевич, 2016 б, 2017 б].

Теорема 2.4. Пусть функиия $F(X)$ удовлетворлет соотношению:

$$
F(X)=\prod_{l=1}^{K} f_{l}\left(y_{l}\right), \quad y_{l}=\sum_{n \in I_{l}} c_{n} x_{n} .
$$

Тогда уравнение (1.1) имеет решение вида

$$
u(X)=U\left(y_{1}, \ldots, y_{K}\right),
$$

причём функиия $U\left(y_{1}, \ldots, y_{K}\right)$ является решением уравнения:

$$
\sum_{k=1}^{K} A_{k} \frac{\partial^{2} U}{\partial y_{k}^{2}}=C g(U) \prod_{k=1}^{K}\left\{f_{k}\left(y_{k}\right)\left(\frac{\partial U}{\partial y_{k}}\right)^{\beta_{\Sigma k}}\right\}
$$

где $C, A_{k}$ определяются выражениями:

$$
C=\prod_{i=1}^{N} c_{i}^{\beta_{i}}, \quad A_{k}=\sum_{i \in I_{k}} a_{i} c_{i}^{2} .
$$

Доказательство. Доказательство проводится аналогично теоремам 2.2, 2.3 путем подстановки функции (2.30) в уравнение (1.1) и последующего преобразования этого уравнения к переменным $y_{k}$, в результате чего получаем уравнение (2.31). Теорема доказана.

Пример. Предположим, что $f_{l}\left(y_{l}\right)=y_{l}^{\alpha_{l}}$ при $\forall l \in \Xi ; A_{l}=0$ при $\forall l \in \Xi, \quad l \neq k$. При указанных предположениях будем искать решение уравнения (2.31) в виде:

$$
U\left(y_{1}, \ldots, y_{K}\right)=V(z), \quad z=\prod_{l=1}^{K} y_{l}^{\sigma_{l}} .
$$

Без ограничения общности положим $\sigma_{k}=1$; остальные показатели $\sigma_{l}$ будут определены ниже. Подставляя (2.32) в (2.31), приходим к уравнению:

$$
A_{k} V^{\prime \prime}(z) \prod_{l=1, l \neq k}^{K} y_{l}^{2 \sigma_{l}}=C g(V) \prod_{l=1}^{K}\left\{f_{l}\left(y_{l}\right)\left(z V^{\prime}(z) \frac{\sigma_{l}}{y_{l}}\right)^{\beta_{\Sigma l}}\right\} .
$$

С помощью элементарных преобразований уравнение (2.33) приводим к виду:

$$
V^{\prime \prime}(z)=B_{k} g(V)\left[z V^{\prime}(z)\right]^{\beta_{\Sigma}} y_{k}^{\alpha_{k}-\beta_{\Sigma k}} \prod_{l=1, l \neq k}^{K} y_{l}^{\alpha_{l}-\beta_{\Sigma l}-2 \sigma_{l}},
$$

где $B_{k}=\frac{C}{A_{k}} \prod_{l=1}^{K} \sigma_{l}^{\beta_{\Sigma l}}$. Для того, чтобы уравнение (2.34) могло быть сведено к ОДУ относительно $V(z)$, должно выполняться условие:

$$
y_{k}^{\alpha_{k}-\beta_{\Sigma k}} \prod_{l=1, l \neq k}^{K} y_{l}^{\alpha_{l}-\beta_{\Sigma l}-2 \sigma_{l}}=z^{\nu} .
$$

Так как в силу сделанного выше предположения $\sigma_{k}=1$, то из (2.35) следует:

$$
\nu=\alpha_{k}-\beta_{\Sigma k}, \quad \nu \sigma_{l}=\alpha_{l}-\beta_{\Sigma l}-2 \sigma_{l} \quad(l \neq k) .
$$


Тогда из (2.36) находим показатели $\sigma_{l}$ :

$$
\sigma_{l}=\frac{\alpha_{l}-\beta_{\Sigma l}}{\alpha_{k}-\beta_{\Sigma k}+2} \quad(l \neq k)
$$

Из (2.34) получаем, что функция $V(z)$ должна удовлетворять следующему ОДу:

$$
V^{\prime \prime}(z)=B_{k} g(V) z^{\beta_{\Sigma}+\nu}\left[V^{\prime}(z)\right]^{\beta_{\Sigma}} .
$$

(2.32) представляет собой решение типа агрегированных бегущих волн [Рахмелевич, 2016б], причём функция $V(z)$ является решением ОДУ (2.38).

Таким образом, в данной работе исследовано неавтономное $\mathrm{N}$-мерное уравнение с частными производными второго порядка, правая часть которого содержит произвольную нелинейность по неизвестной функции и нелинейности степенного типа по её первым производным. Найдено решение этого уравнения типа бегущей волны в неявном виде. Для случая степенной нелинейности по неизвестной функции получены явные решения типа бегущей волны, в частности, в виде степенной, экспоненциальной и логарифмической функций. Проанализировано, как влияют параметры уравнения на свойства найденных решений.

\section{Список литературы}

1. Кудряшов Н. А. 2010. Методы нелинейной математической физики. Долгопрудный: Изд-во «Интеллект», 368.

2. Полянин А. Д., Зайцев В. Ф. 2002. Справочник по нелинейным уравнениям математической физики: точные решения. М.: Физматлит, 432.

3. Полянин А. Д., Зайцев В. Ф., ЖКуров А. И. 2005. Методы решения нелинейных уравнений математической физики и механики. М.: Физматлит, 256.

4. Рахмелевич И. В. 2016 а. О редукции многомерных уравнений первого порядка с мультиоднородной функцией от производных. Известия вузов. Математика, 4: 57-67.

5. Рахмелевич И. В. 2016 б. О решениях типа агрегированных бегущих волн для линейных уравнений в частных производных с переменными коэффициентами. Научные ведомости Белгородского университета. Математика. Физика, 43 (13): 30-38.

6. Рахмелевич И. В. 2017 а. О псевдополиномиальных решениях двумерного уравнения, содержащего произведение частньх производных. Научные ведомости Белгородского университета. Математика. Физика, 47 (13): 45-50.

7. Рахмелевич И. В. 2017 б. О многомерных уравнениях в частных производных со степенными нелинейностями по первым производным. Уфимский математический журнал, 9 (1): 98-109.

8. Рахмелевич И.В. 2017 в. Двумерное эллиптическое уравнение с нелинейным источником, содержащим степени первых производных. Научные ведомости Белгородского университета. Математика. Физика. 49 (27): 33-41.

9. Рахмелевич И. В. 2018. Многомерное неавтономное уравнение, содержащее произведение степеней частных производных. Вестник Санкт-Петербургского государственного университета: Математика, механика, астрономия. 5(63), № 1: 119-130.

10. Grundland A.M., Infeld E. 1992. A family of non-linear Klein - Gordon equations and their solutions. Journal of Mathematical Physics, 33 (7): 2498-2503.

11. Miller J. (Jr.), Rubel L.A. 1993. Functional separation of variables for Laplace equations in two dimensions. Journal of Physics A, 26: 1901-1913.

12. Polyanin A. D. 2019 a. Construction of exact solutions in implicit form for PDEs: New functional separable solutions of non-linear reaction-diffusion equations with variable coefficients. International Journal of Non-Linear Mechanics, 111: 95-105.

13. Polyanin A. D. 2019 b. Comparison of the effectiveness of different methods for constructing exact solutions to nonlinear PDEs. Generalizations and new solutions. Mathematics, 7(5): 386 . DOI $10.3390 /$ math7050386. 
14. Zhdanov R. Z. 1994. Separation of variables in the non-linear wave equation. Journal of Physics A, 27: L291-L297.

\section{References}

1. Kudryashov N. A. 2010. Metody nelineynoy matematicheskoy fiziki [Methods of nonlinear mathematical physics]. Dolgoprudny: Publ. «Intellect», 368.

2. Polyanin A. D., Zaytsev V. F. 2012. Handbook of Nonlinear Partial Differential Equations, 2nd Edition. Boca Raton - London: Chapman and Hall - CRC Press, 1912 (in Russian).

3. Polyanin A. D., Zaytsev V. F., Zhurov A. I. 2005. Metody resheniya nelineynykh uravneniy matematicheskoy fiziki i mekhaniki [Methods of solving of nonlinear equations of mathematical physics and mechanics] M: Fizmatlit, 256.

4. Rakhmelevich I. V. 2016 a. Reduction of multidimensional first order equations with multi-homogeneous function of derivatives. Russian Mathematics. 60 (4): 47-55 (in Russian).

5. Rakhmelevich I. V. 2016 b. O resheniyakh tipa agregirovannykh begushikh voln dlya lineynykh uravneniy v chastnykh proizvodnykh s peremennymi koefficientami [On the solutions type of aggregated travelling waves for linear partial differential equations with variable coefficients] Belgorod State University Scientific Bulletin. Mathematics. Physics, 43 (13): 30-38.

6. Rakhmelevich I. V. 2017 a. O psevdopolynomialnykh resheniyakh dvumernogo uravneniya, soderzhashego proizvedenie chastnykh proizvodnykh [On the pseudo-polynomial solutions of two-dimensional equati-on containing the production of partial derivatives] Belgorod State University Scientific Bulletin. Mathematics. Physics, 47 (13): 45-50.

7. Rakhmelevich I. V. 2016 c. On multi-dimensional partial differential equations with power nonlinearities in first derivatives. Ufa Mathematical Journal, 8 (4): 98-108 (in Russian).

8. Rakhmelevich I. V. 2017 b. Dvumernoe ellipticheskoe uravnenie s nelineynym istochnikom, soderzhashim stepeni pervykh proizvodnykh [Two-dimensional elliptic equation with nonlinear source containing powers of first derivatives] Belgorod State University Scientific Bulletin. Mathematics. Physics, 49 (27): 33-41.

9. Rakhmelevich I. V. 2018. Mnogomernoe neavtonomnoe uravnenie, soderzhashee proizvedenie stepeney chastnykh proizvodnykh [Multi-dimensional non-autonomous equation contaning the production of powers of partial derivatives] Vestnik SpBSU. Mathematics, mechanics,astronomy, 5(63), No 1: 119130 .

10. Grundland A. M., Infeld E. 1992. A family of non-linear Klein - Gordon equations and their solutions. Journal of Mathematical Physics, 33 (7): 2498-2503.

11. Miller J. (Jr.), Rubel L. A. 1993. Functional separation of variables for Laplace equations in two dimensions. Journal of Physics A, 26: 1901-1913.

12. Polyanin A. D. 2019 a. Construction of exact solutions in implicit form for PDEs: New functional separable solutions of non-linear reaction-diffusion equations with variable coefficients. International Journal of Non-Linear Mechanics, 111: 95-105.

13. Polyanin A. D. 2019 b. Comparison of the effectiveness of different methods for constructing exact solutions to nonlinear PDEs. Generalizations and new solutions. Mathematics, 7(5): 386 . DOI $10.3390 /$ math7050386.

14. Zhdanov R. Z. 1994. Separation of variables in the non-linear wave equation. Journal of Physics A, 27: L291-L297.

Получена 12.05.2020

Рахмелевич Игорь Владимирович - кандидат технических наук, доцент, доцент кафедры математических и естественнонаучных дисциплин Нижегородского государственного университета им. Н. И. Лобачевского пр. Гагарина, 23, г. Нижний Новгород, Россия, E-mail: igor-kitpd@yandex.ru 